Original

\title{
Immunohistochemical analysis of p53 and PCNA expression in calcifying odontogenic cyst
}

\author{
Shadi Saghafi ${ }^{1)}$, Reza Zare-Mahmoodabadi ${ }^{1)}$, Jahanshah Salehinejad ${ }^{1)}$, \\ Hamideh Kadeh ${ }^{1)}$ and Monavar Afzal-Aghaee ${ }^{2)}$ \\ ${ }^{1)}$ Department of Oral and Maxillofacial Pathology, Dental Research Center, Faculty of Dentistry, \\ Mashhad University of Medical Sciences, Mashhad, Iran \\ ${ }^{2}$ Social Medicine Specialist, Mashhad University of Medical Sciences, Mashhad, Iran
}

(Received 21 July and accepted 20 October 2010)

\begin{abstract}
Calcifying odontogenic cyst (COC) is a developmental odontogenic cyst in the jaw. Because of its diverse histopathologic features and biological behavior, there has long been confusion with regard to its nature as a cyst or neoplasm. This study evaluated the proliferative activity of $57 \mathrm{COC}$ samples, including simple cyst (10 cases), cystic neoplasm ( 34 cases), solid neoplasm ( 6 cases) and combined lesion ( 7 cases) by p53 and PCNA immunohistochemical staining. For assessment of p53 and PCNA positivity, the number of positively stained cells with brown-stained nuclei was counted in 1000 cells from each sample. p53 and PCNA expression in the solid neoplasm subtype were significantly higher when compared to cystic neoplasm and simple cyst $(P<0.05)$. The lowest p53 and PCNA expression was found in the simple cyst subtype. p53 and PCNA expression in the basal and suprabasal layers was significantly higher in the solid subtype when compared to others, and the difference between COC groups was significant. The results demonstrated that within benign types of $\mathrm{COC}$, the amount of p53 and PCNA in proliferative epithelium is significantly higher when compared to non-proliferative epithelium. p53 and PCNA markers are possible parameters for differentiation of COC subtypes. (J Oral Sci 52, 609-
\end{abstract}

Correspondence to Dr. Jahanshah Salehinejad, Department of Oral and Maxillofacial Pathology, Dental Research Center, Faculty of Dentistry, Mashhad University of Medical Sciences, Vakilabad Blv., P. O. Box 911735-984, Mashhad, Iran

Tel: +98-511-8829501

Fax: +98-511-8829500

Email: j_salehinejad@yahoo.com
613, 2010)

Keywords: calcifying odontogenic cyst; p53; PCNA; immunohistochemistry.

\section{Introduction}

Calcifying odontogenic cyst (COC) is a developmental odontogenic cyst that represents approximately $1 \%$ of jaw cysts (1). COC was first described by Rywkind in 1932 (2), but it was Gorlin, in 1962, who defined this lesion as a separate entity from so-called calcifying odontogenic tumor (3). COC exhibits considerable diversity in clinical and histopathological features, as well as biological behavior $(1,4)$, and several classifications for this lesion have been proposed. In 1981, Praetorius et al. assigned COC into two groups, a cyst and a neoplasm and classified it into four different histologic patterns (5):

1. Simple unicystic

2. Odontoma associated

3. Ameloblastomatous proliferating

4. Dentinogenic ghost cell tumor

In 1992, the World Health Organization (WHO) classified $\mathrm{COC}$ as a neoplasm rather than a cyst, but confirmed that most cases are neoplastic (6). In 1998, Toida suggested a classification that included three main subdivisions of COC: cystic, neoplastic and combined lesions. The former lesions have a truly cystic architecture and are known as calcifying ghost cell odontogenic cyst (CGCOC). On the other hand, neoplastic lesions are divided into benign and malignant types. The benign neoplasm is known as calcifying ghost cell odontogenic tumor (CGCOT), and can 
appear to be either cystic or solid. Cystic CGCOT shows cystic architecture and an intramural ameloblastoma-like proliferation, while solid CGCOT has a growth pattern that is consistent with a neoplasm, and is characterized by ameloblastoma-like proliferation that infiltrates the connective tissue with varying amounts of ghost cells and dentinoid structures. When CGCOC and CGCOT are associated with other types of odontogenic lesions, including odontoma and ameloblastoma, they are listed as combined lesions (Table 1) (4). Consequently in 2005, WHO described the cystic type of COC as a low-grade neoplasm, renaming it as calcifying cystic odontogenic tumor (CCOT), and renamed the solid type of COC as dentinogenic ghost cell tumor (DGCT), which is a more aggressive entity $(7,8)$.

Proliferating cell nuclear antigen (PCNA) is a nuclear non-histone protein necessary for DNA synthesis and is an accessory protein for DNA polymerase-alpha, which is elevated during the G1/S phase of the cell cycle (9). Assessment of PCNA is one of the most common immunohistochemical methods to assess the state of cellular proliferation. The incidence of PCNA expression correlates to mitotic activity (10). TP53 is a tumor suppressor gene situated on chromosome 17p13. In normal cells, it regulates proliferation and its inactivity leads to neoplastic changes. Activation of p53 protein, a product of TP53 after DNA damage, is an important protective mechanism that induces either G1/S cell-cycle arrest to allow DNA repair or apoptosis if DNA has suffered irreversible damage. Mutation and loss of heterozygosity of TP53 is associated with increased cellular proliferation $(11,12)$.

The purpose of the present study was to assess p53 and PCNA expression in various types of calcifying odontogenic cyst by immunohistochemical staining.

\section{Materials and Methods}

Fifty-seven cases of COC were retrieved from the archive of the Oral and Maxillofacial Pathology Department of Mashhad Dental School, Iran. Histological sections stained with hematoxylin-eosin (H\&E) were reviewed by two pathologists and subclassified according to the histological hallmarks described by Toida (13). Toida's classification was used in the present study because it is both simple and practical. For immunohistochemical staining, paraffinembedded tissue that had been fixed in $10 \%$ buffered formalin was cut into $4-\mu \mathrm{m}$ sections, which were then mounted on silicon-coated glass slides and air dried at room temperature. Sections were deparaffinized in xylene, rehydrated using a graded alcohol series, and immersed in $0.3 \%$ hydrogen peroxide in methanol in order to block endogenous peroxidase. Sections were then washed in phosphate buffered saline (PBS). Antigen retrieval was performed for $20 \mathrm{~min}$ in a water bath using a low-pH retrieval solution, and the sections were then incubated in the same solution for $45 \mathrm{~min}$ to reach room temperature. Monoclonal p53 (clone DO-7, DAKO, California, USA) and PCNA (clone PC10, DAKO) antibodies were used according to the manufacturer's instructions. Sections were rinsed, counterstained with Mayer hematoxylin, rehydrated and cleared in xylene, and slides were then mounted in Permount. Sections of colon adenocarcinoma and reactive lymph nodes were used as positive controls for p53 and PCNA respectively, and as a negative control, primary antibody was omitted. All slides were separately observed by two pathologists.

For assessment of p53 and PCNA positivity, the number of positive-stained cells was counted in 1000 cells of each sample at $\times 400$ magnification; 500 cells in the basal layer and 500 cells in the suprabasal layer, as described by Oliveira (9). The percentage of positive cells, epithelial cells with brown-stained nuclei, was then calculated.

Statistical analysis was performed by ANOVA and Kruskal Wallis tests using SPSS 15 software. Statistical significance was set at $P<0.05$.

\section{Results}

The 57 cases of COC studied included 36 males and 21 females. Mean age of patients was 27.1 years (range, 662 years). Forty cases were in the mandible and 17 cases were in the maxilla. COC subtypes were classified in

Table 1 Subtyping of calcifying ghost cell odontogenic cyst, as proposed by Toida (4)

\begin{tabular}{|c|c|}
\hline Lesion & Subtype \\
\hline Cyst & Calcifying ghost cell odontogenic cyst(CGCOC) \\
\hline \multicolumn{2}{|l|}{ Neoplasm } \\
\hline Benign & Calcifying ghost cell odontogenic tumor(CGCOT) \\
\hline Cystic & Cystic CGCOT \\
\hline Solid & Solid CGCOT \\
\hline Malignant & Malignant CGCOT \\
\hline Combined lesions & $\begin{array}{l}\text { CGCOC, CGCOT and malignant CGCOT, associated with other } \\
\text { odontogenic lesions, such as odontoma and ameloblastoma }\end{array}$ \\
\hline
\end{tabular}


accordance with Toida as simple cyst (CGCOC) (10 cases), cystic neoplasm (cystic CGCOT) (34 cases), solid neoplasm (solid CGCOT) (6 cases) and combined lesions, which were cystic type lesions associated with odontoma (7 cases). None of the present cases were malignant. All COC cases showed p53 and PCNA reactivity in both basal and suprabasal layers, with the amounts decreasing gradually from the basal to the suprabasal layer (Figs. 1-4).

p53 and PCNA expression in the solid neoplasm subtype was significantly higher than in the cystic neoplasm and simple cystic subtypes $(P<0.05)$. However, the lowest p53 and PCNA expression was seen in the simple cystic subtype

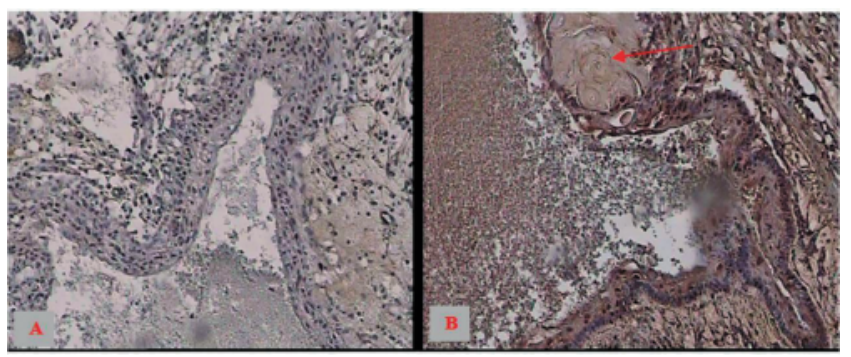

Fig. 1 Photomicrograph showing odontogenic epithelium in the simple cystic subtype of COC. A: p53 expression, B: PCNA expression (arrows indicate ghost cells) (original magnification, $\times 100$ ).

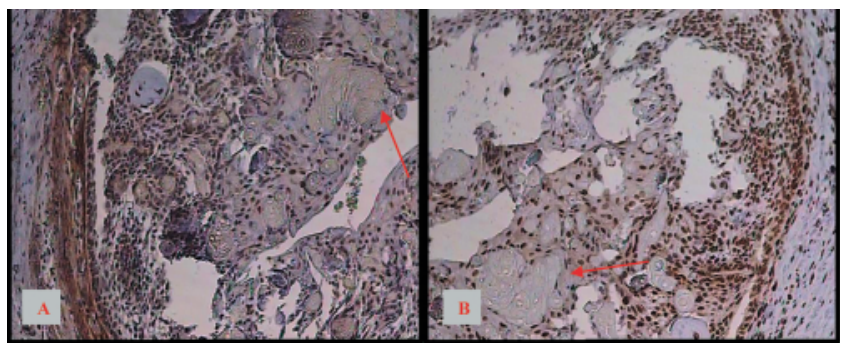

Fig. 3 Photomicrograph showing odontogenic epithelium in the solid neoplastic subtype of COC. A: p53 expression, B: PCNA expression (arrows indicate ghost cells) (original magnification, $\times 100$ ).
(Table 2). From one-way ANOVA, significant differences were seen between the study groups for both and p53 expression $(P=0.001)$ and PCNA expression $(P=0.024)$.

From analysis of p53 expression in the basal and suprabasal layers, the solid neoplasm subtype showed higher p53 expression. From the Kruskal Wallis test, there was a significant difference between COC groups $(P=$ 0.003 in basal layer; $P=0.002$ in suprabasal layer). PCNA expression in the basal and suprabasal layers was significantly higher in the solid subtype than in the other subtypes $(P=0.003)$. (Table 3 )

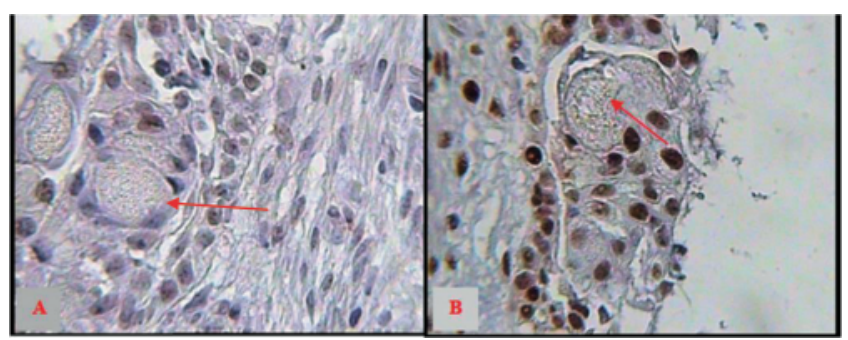

Fig. 2 Photomicrograph showing odontogenic epithelium in the neoplastic cystic subtype of COC. A: p53 expression, B: PCNA expression (arrows indicate ghost cells) (original magnification, $\times 400)$.

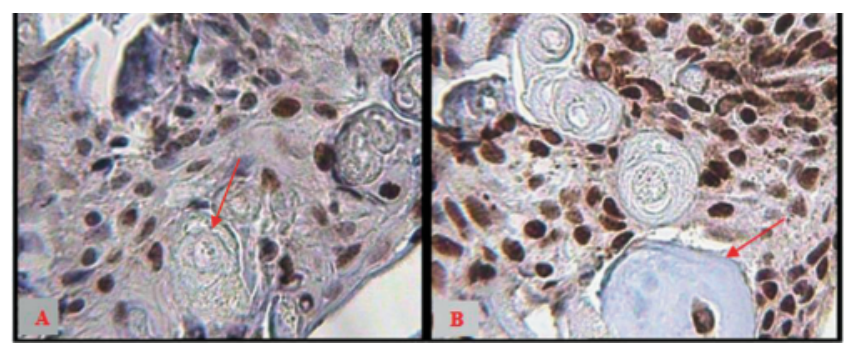

Fig. 4 Photomicrograph showing odontogenic epithelium in the combined subtype of COC. A: p53 expression, B: PCNA expression (arrows indicate calcification) (original magnification, $\times 400$ ).

Table 2 Mean percentage of cells positive for p53 and PCNA in each subtype of $\mathrm{COC}$

\begin{tabular}{llllll}
\hline Lesion & N & \multicolumn{2}{c}{ P53+ } & \multicolumn{2}{c}{ PCNA+ } \\
& & Mean\% $\mathbf{\text { SD }}$ & Range & Mean\% $\mathbf{\text { SD }}$ & Range \\
\hline Simple cyst & 10 & $22.8 \pm 12.19$ & $14-55$ & $64.9 \pm 10.42$ & $45-75$ \\
Cystic neoplasm & 34 & $34.58 \pm 16.36$ & $8-75$ & $69.91 \pm 13.95$ & $40-90$ \\
Solid neoplasm & 6 & $54.33 \pm 10.96$ & $40-68$ & $86.16 \pm 7.9$ & $80-99$ \\
Combined lesion & 7 & $25 \pm 10.29$ & $15-38$ & $71.85 \pm 16.43$ & $41-85$ \\
Total & 57 & $33.42 \pm 16.74$ & $8-75$ & $70.98 \pm 14.09$ & $40-99$ \\
\hline
\end{tabular}


Table 3 Mean percentage of cells positive for p53 and PCNA in basal and suprabasal layers in each subtype of COC

\begin{tabular}{|c|c|c|c|c|c|c|}
\hline \multirow{2}{*}{ Lesion } & \multirow{2}{*}{$\mathbf{N}$} & \multirow{2}{*}{ Layer } & \multicolumn{2}{|c|}{ P53+ } & \multicolumn{2}{|c|}{ PCNA +} \\
\hline & & & Mean\% & SD & Mean \% & SD \\
\hline \multirow{2}{*}{ Simple cyst } & \multirow{2}{*}{10} & Basal & 16.90 & 4.97 & 54.60 & 8.32 \\
\hline & & Suprabasal & 13.30 & 4.64 & 50.50 & 8.79 \\
\hline \multirow{2}{*}{ Cystic neoplasm } & \multirow{2}{*}{34} & Basal & 24.70 & 14.10 & 63.02 & 12.50 \\
\hline & & Suprabasal & 22.1 & 13.90 & 57.64 & 13.4 \\
\hline \multirow{2}{*}{ Solid neoplasm } & \multirow{2}{*}{6} & Basal & 43 & 16.23 & 77.83 & 4.16 \\
\hline & & Suprabasal & 39.50 & 16.69 & 74.50 & 4.08 \\
\hline \multirow{2}{*}{ Combined lesion } & \multirow{2}{*}{7} & Basal & 15 & 8.69 & 65.71 & 12.25 \\
\hline & & Suprabasal & 12.28 & 8.49 & 61.14 & 12.54 \\
\hline \multirow{2}{*}{ Total } & \multirow{2}{*}{57} & Basal & 24.08 & 14.52 & 63.43 & 12.56 \\
\hline & & Suprabasal & 21.22 & 14.40 & 58.59 & 13.30 \\
\hline
\end{tabular}

\section{Discussion}

COC represents approximately $5-7 \%$ of odontogenic tumors and $1 \%$ of all cysts of the jaw $(1,6)$. Most cases $(70 \%)$ are intraosseous lesions almost equally affecting the maxilla and mandible, with a predilection to the anterior regions $(2,6)$. It mainly affects adults in their third to fourth decade with no gender predilection. COC is equally distributed between the two jaws, commonly seen anterior to the first molar region $(2,6)$, and is a unique lesion possessing both cystic and neoplastic potential (2). Some authors have considered $\mathrm{COC}$ to be a tumor with a tendency for cystic formation and designated it ghost cell odontogenic tumor. WHO described COC as a non-neoplastic cyst, but classified it as a tumor (14).

Histologically, the epithelial lining of COC shows a basal layer of ameloblast-like columnar cells, and the overlying layer comprises loosely arranged epithelial cells resembling stellate reticulum, with few or masses of ghost epithelial cells that may be in the epithelial cell lining or in the fibrous capsule. The ghost epithelial cells may also undergo calcification. Dentinoid material may be seen adjacent to the epithelium in connective tissue $(1,2,6,14,15)$. In neoplastic $\mathrm{COC}$, luminal or mural proliferations that have an ameloblastomatous appearance can be seen (6). In addition, COC may be present with odontoma or other odontogenic tumors, such as ameloblastic fibroma, ameloblastic fibroodontoma, ameloblastoma and adenomatoid odontogenic tumor (1).

Toida suggested a classification that included three main subdivisions of COC: cystic, neoplastic and combined lesions (4). Although it is difficult to distinguish cystic COC from the cystic variant of neoplastic $\mathrm{COC}$, this classification is more practical and correlates reasonably well with COC proliferation activity. We therefore used this classification system in this study.

The results of the present study suggest that the expression of PCNA antigen in the neoplasm group is significantly higher than in the simple cystic group. It was also confirmed that within the neoplasm group, the amount of PCNA was greater in the solid subtype than in the cystic subtype. Therefore, PCNA can be effectively used to distinguish different subtypes of COC, particularly in the case of solid neoplasm, and the present results corroborate those of previous studies.

Fregnani et al. (2003) found that the mean PCNA and Ki-67 labeling indexes are correlated with epithelial proliferation. In addition, proliferative activity was greater in the epithelium of the central cystic COC, particularly when associated with odontoma and peripheral lesions. They concluded that immunohistochemical methods may be useful in diagnosing variants of COC (6). Takata (10) found that the mean PCNA labeling index in the nonproliferative type was lower than that in the proliferative type, and that in the malignant $\mathrm{COC}$ was higher than in benign COC. Yoshida et al. (16) also found that the mean Ki-67 labeling index was slightly greater in proliferative $\mathrm{COC}$, combined $\mathrm{COC}$ or $\mathrm{COC}$ with ameloblastomatous proliferation than in those without these histological features, and that the proportion of cases positive for bcl2 was slightly higher in COCs with odontoma than in those without odontoma.

The mean amount of p53 antigen observed in the solid neoplasm group was the highest, and when compared to other groups, this difference was significant. p53 is therefore a useful marker to distinguish between different subtypes of COC. Piattelli (17) reported a case of dentinogenic ghost cell tumor and the immunohistochemical analysis for Mib-1 and bcl-2 showed strong positivity in the odontogenic epithelium, while positivity for p53 was rarely observed. Özveren (12) reported high concentrations of p53 protein in odontogenic lesions. Oliveira et al. (9) analyzed patterns of p53 and PCNA expression in 
odontogenic cysts and found that COC had elevated values of both PCNA and p53 expression, and there was a direct and significant correlation between the two markers in the two layers studied. Therefore, p53 may be associated with the rate of proliferation in these lesions. Motosugi et al. (18) also showed that Ki-67 labeling index and p53 positivity were higher in ghost cell odontogenic carcinoma derived from COC.

Within benign types of COC, the amount of observed p53 and PCNA in the proliferative epithelium is significantly higher than that in non-proliferative epithelium. To our knowledge, this is the first study to analyze p53 expression in COC subtypes.

\section{Acknowledgments}

This study was supported by a grant (No.87070) from the Vice Chancellor of Mashhad University of Medical Sciences, Mashhad, Iran.

\section{References}

1. Kamboj M, Juneja M (2007) Ameloblastomatous Gorlin's cyst. J Oral Sci 49, 319-323.

2. Rajkumar K, Kamal K, Sathish MR, Leena S (2004) Calcifying odontogenic cyst. J Oral Maxillofac Pathol 8, 99-103.

3. Gong Y, Wang L, Wang H, Li T, Chen X (2009) The expression of NF- $\mathrm{KB}, \mathrm{Ki}-67$ and MMP-9 in CCOT, DGCT and GCOC. Oral Oncol 45, 515-520.

4. Toida M (1998) So-called calcifying odontogenic cyst: review and discussion on the terminology and classification. J Oral Pathol Med 27, 49-52.

5. Praetorius F, Hjørting-Hansen E, Gorlin RJ, Vickers RA (1981) Calcifying odontogenic cyst. Range, variations and neoplastic potential. Acta Odontol Scand 39, 227-240.

6. Fregnani ER, Pires FR, Quezada RD, Shih IeM,Vargas PA, de Ameida OP (2003) Calcifying odontogenic cyst: clinicopathological features and immunohistochemical profile of 10 cases. J Oral Pathol Med 32, 163-170.

7. Ahn SG, Kim SA, Kim SG, Lee SH, Kim J, Yoon $\mathrm{JH}$ (2008) $\beta$-catenin gene alterations in a variety of so-called calcifying odontogenic cysts. APMIS 116 , 206-211.

8. Ledesma-Montes C, Gorlin RJ, Shear M, Prae Torius F, Mosqueda-Taylor A, Altini M, Unni K, Paes de Almeida O, Carlos-Bregni R, Romero de León E, Phillips V, Delgado-Azañero W, Meneses-García A
(2008) International collaborative study on ghost cell odontogenic tumours: calcifying cystic odontogenic tumour, dentinogenic ghost cell tumour and ghost cell odontogenic carcinoma. J Oral Pathol Med 37, 302-308.

9. de Oliveira MG, Lauxen Ida S, Chaves AC, Rados PV, Sant'Ana Filho M (2008) Immunohistochemical analysis of the patterns of p53 and PCNA expression in odontogenic cystic lesions. Med Oral Patol Oral Cir Bucal 13, E275-280.

10. Takata T, Lu Y, Ogawa I, Zhao M, Zhou ZY, Mock D, Nikai H (1998) Proliferative activity of calcifying odontogenic cysts as evaluated by proliferating cell nuclear antigen labeling index. Pathol Int 48, 877881.

11. Kumamoto H, Izutsu T, Ohki K, Takahashi N, Ooya $\mathrm{K}$ (2004) p53 gene status and expression of p53, $\mathrm{MDM} 2$, and $\mathrm{p} 14^{\mathrm{ARF}}$ proteins in ameloblastomas. $\mathrm{J}$ Oral Pathol Med 33, 292-299.

12. Özveren A, Tuskan C, Yaltirik M, Atalay B, Erseven G (2003) Expression of the tumour suppressor gene p53 in odontogenic cysts. Turk J Med Sci 33, 243247.

13. Toida M (2000) Proliferative activity and subtyping of calcifying odontogenic cyst. Pathol Int 50, 8183.

14. Moleri AB, Moreira LC, Carvalho JJ (2002) Comparative morphology of 7 new cases of calcifying odontogenic cysts. J Oral Maxillofac Surg 60, 689-696.

15. Folpe AL, Tsue T, Rogerson L, Weymuller E, Oda D, True LD (1998) Odontogenic ghost cell carcinoma: a case report with immunohistochemical and ultrastructural characterization. J Oral Pathol Med 27, 185-189.

16. Yoshida M, Kumamoto H, Ooya K, Mayanagi H (2001) Histopathological and immunohistochemical analysis of calcifying odontogenic cysts. J Oral Pathol Med 30, 582-588.

17. Piattelli A, Fioroni M, Alberti L, Rubini C (1998) Immunohistochemical analysis of a dentogenic ghost cell tumour. Oral Oncol 34, 502-507.

18. Motosugi U, Ogawa I, Yoda T, Abe T, Sugasawa M, Murata S, Yasuda M, Sakurai T, Shimizu Y, Shimizu M (2009) Ghost cell odontogenic carcinoma arising in calcifying odontogenic cyst. Ann Diagn Pathol 13, 394-397. 